\title{
Quantitative Phase Analysis of Complex Fats during Crystallization
}

\author{
Naomi Arita-Merino, Hein van Valenberg, Elliot Paul Gilbert, and Elke Scholten* \\ Cite This: Cryst. Growth Des. 2020, 20, 5193-5202 \\ Read Online
}

ABSTRACT: The quantitative study of polymorphism in fats is complicated due to their complex composition and consequent intricate crystallization behavior. We aimed to illustrate how quantitative phase analysis can be performed in crystallizing complex fats. The advantages of our proposed method are that detailed structural information or internal standards are not needed and that it can be applied even to wide-angle X-ray diffraction patterns with a high noncrystalline (liquid fat) contribution and overlapping peaks. Diffraction patterns of palm oil and anhydrous milk fat during crystallization were decomposed by fitting a model based on Pearson type VII functions. From the decomposed

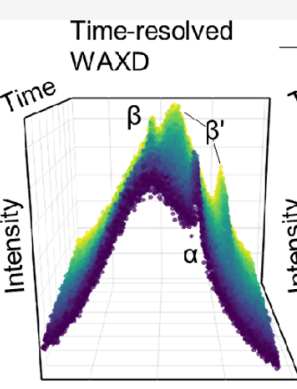

q

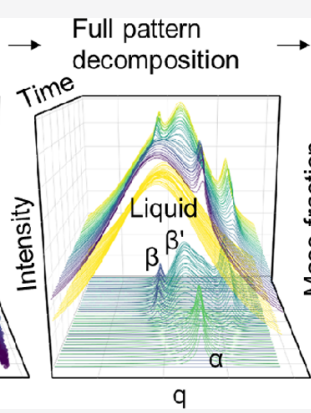

Phase transition diagram

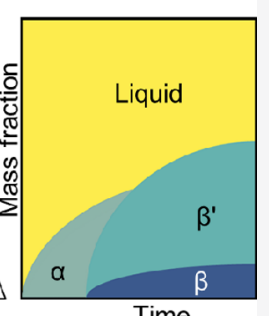

Time patterns, the contribution of each phase was quantified as a function of time. Each contribution to the pattern was then converted to its mass fraction using response factors derived from the corresponding solid fat content, which was measured using low-resolution nuclear magnetic resonance (NMR). This approach allowed us to quantify the evolution of liquid and polymorphic phases during the early crystallization of complex fats and, for the first time, to quantify coexisting $\alpha, \beta^{\prime}$, and $\beta$ phases. This method is flexible enough for fats with diverse triacylglycerol profiles yet detailed enough to quantify phase transitions in a system as complex as milk fat.

\section{INTRODUCTION}

The functional and sensory properties of numerous products, such as the spreadability and mouthfeel of a margarine, rely on the crystallization behavior of fats. Among the many factors involved in fat crystallization, polymorphism plays a key role. Therefore, the study of the polymorphic structures in fats is fundamental to understand, predict, and to some extent control fat crystallization. A fat can form alternative crystal structures known as polymorphs, in which the long hydrocarbon chains are packed differently into a crystal lattice. ${ }^{1}$ Although other forms have been reported, the polymorphic structures most commonly formed in fats are $\alpha, \beta^{\prime}$, and $\beta$, in increasing order of stability and packing density. ${ }^{2}$ These polymorphs have characteristic short spacings between the fatty acid acyl chains that result in specific peaks in an X-ray diffraction (XRD) pattern. ${ }^{3,4}$ In the wide-angle region, these Xray diffraction peaks make it possible to identify the different polymorphs even in fats with a complex composition. However, quantification of the different fat polymorphic phases is not straightforward, especially in fats with complex composition.

The diverse composition of most fats, reflected in their complex crystallization behavior, obstructs the use of common quantitative phase analysis (QPA) methods. Although a few methods ${ }^{5-7}$ have been developed for phase quantification in complex fats, XRD is still used predominantly in a qualitative manner, not for phase quantification. As will be further discussed, previous approaches have merits and yield reasonable results. However, there is a need for a QPA method that can quantify coexisting liquid $\alpha, \beta^{\prime}$, and $\beta$ polymorphs in complex fats on the basis of data obtained from easily accessible techniques such as in-house XRD.

For any material, including fats, there are two essential components to perform QPA on the basis of XRD patterns. First, the contribution to the pattern of any phase present must be quantified separately, and second, these contributions to the pattern need to be related to the mass fractions of each of the respective phases. In the case of complex fats, both steps are complicated.

Fats, especially nonmodified animal and vegetable fats, are composed of a wide variety of triacylglycerols (TAGs), which leads to complex XRD patterns; quantification of different phases becomes therefore complicated. Typically, edible fats can contain more than 500 different TAGs. ${ }^{1}$ With such a heterogeneous composition, noncrystalline (liquid) and crystalline phases can coexist over wide temperature ranges. ${ }^{1}$ Also, two or more crystalline polymorphic phases can coexist during transition periods. ${ }^{1}$ Although TAGs are monotropic materials, meaning that one of their polymorphic forms is

Received: March 26, 2020

Revised: June 17, 2020

Published: June 18, 2020 
thermodynamically the most stable, liquid and different polymorphic phases can coexist also for longer periods of time, especially for fats with a widely varying TAG composition. ${ }^{8}$ For example, milk fat (MF) is composed of more than 223 TAG species with different molecular weights, structures, and degrees of unsaturation, all in concentrations below $5 \mathrm{~mol} \%{ }^{9}$. Because of this diversity, MF has a melting range spanning from -40 to $+40{ }^{\circ} \mathrm{C}^{10}$ and can exhibit pseudostable mixtures of different polymorphic phases. At 4 ${ }^{\circ} \mathrm{C}$, liquid fat, $\alpha, \beta^{\prime}$, and $\beta$ can coexist even after 5 days of crystallization. ${ }^{11}$ As the total XRD pattern of a complex fat, for example that presented in Figure 1, is the combined result of a

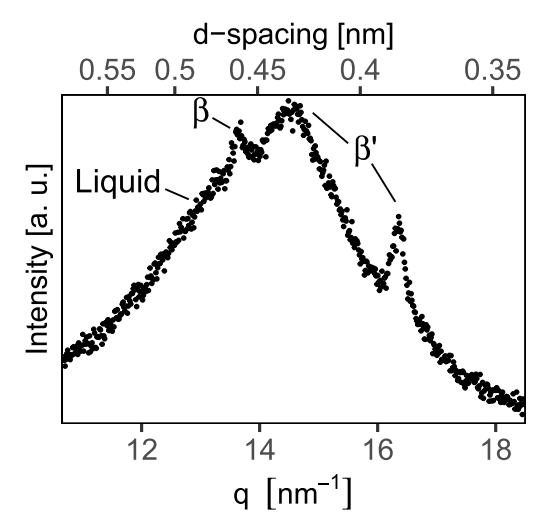

Figure 1. XRD pattern of anhydrous milk fat recorded after $1 \mathrm{~h}$ at 17 ${ }^{\circ} \mathrm{C}$.

liquid phase and many crystallites with diverse TAG composition, size, and polymorphism, the peaks of the different crystalline polymorphs often overlap with each other and with a broad liquid peak, making pattern decomposition more challenging.

To quantify the contribution of the different liquid and polymorphic phases to the pattern, the studies performing QPA in complex fats take one of two approaches. In the first approach, ${ }^{6}$ a single reference peak is selected to characterize the liquid and each one of the polymorphic phases observed in a set of time-resolved wide-angle X-ray diffraction (WAXD) scans. The area of the liquid peak is used as a measure of the liquid fraction, but the relative abundances of coexisting $\alpha$ and $\beta^{\prime}$ phases are estimated according to the heights of their reference peaks in the crystalline patterns. As only the height of a crystalline peak per phase is used in this estimation, calculations are fast and simple. However, accurate quantification of the intensity of a peak should be based on its area rather than its height, ${ }^{12}$ as instrumental and specimen factors can broaden peaks. Furthermore, single-peak approaches can result in phase quantification errors that could be minimized by considering the full pattern instead. ${ }^{13}$ The second approach $^{5,7}$ quantifies peak areas, but instead of using XRD patterns from the wide-angle region where lateral short spacings are measured, it uses small-angle X-ray scattering (SAXS) patterns. From SAXS patterns, phases with different thicknesses of their stacked TAGs (long spacings) can be quantified, and therefore $\alpha$ and $\beta^{\prime}$ phases can be separated as they have distinct layer thicknesses. However, in many cases, this approach cannot differentiate between $\beta^{\prime}$ and $\beta$ phases, as they often show the same long-spacing peak. ${ }^{14-17}$ In those cases, the use of XRD in the wide-angle region is the only alternative to quantify coexisting $\beta^{\prime}$ and $\beta$ phases.
The second step to QPA, the conversion of the contribution of each phase to the diffraction pattern to its mass fraction, requires specific proportionality or response factors for the different liquid and crystalline phases. The response factor of a phase arises from its characteristic structure; therefore, it must be determined for each phase separately, either theoretically or experimentally. The phase-specific response factor $Z M V$ (where $Z, M$, and $V$ are the number of formula units per unit cell, the mass of the formula unit, and the unit cell volume, respectively) can be theoretically derived from structural information in a Rietveld analysis. ${ }^{13}$ Rietveld analysis can be used for phase quantification of multicomponent crystalline materials with known structures. For systems composed of unknown or partially known structures, including noncrystalline components (amorphous or liquid), response factors cannot be theoretically derived. Other quantification methods have been developed for such systems with missing structural information and a high amorphous contribution. In these methods, the response factors are determined experimentally with an internal standard, thus adding a specific component in a known amount. ${ }^{18}$ However, this approach is not ideal to study fat crystallization, as the addition of an external component may alter the crystallization process and the phase composition.

In simple fats, the theoretical derivation of response factors has been carried out. Recently, Calligaris et al. ${ }^{19}$ successfully used the Rietveld method to quantify $\beta^{\prime}$ and $\beta$ phases in binary blends of fully hydrogenated fats. These blends had a simple composition and a negligible liquid contribution. As the individual fats composing the blends displayed only one stable polymorph each, it was possible to perform a Rietveld analysis following the "fundamental parameters approach" (FPA), on the basis of already resolved $\beta^{\prime}$ and $\beta$ structures obtained for single TAGs. ${ }^{20,21}$ Such an approach works for simple fats, but for most commonly used fats, their complex composition makes the approach nonviable. In addition, the specific atomic structure has not been determined yet for some polymorphs, including $\alpha$, making full analysis challenging. For studies of more complex fats, in some of the quantification methods discussed above, the ratio between the areas or heights of the peaks is arbitrarily assumed to be proportional to the ratio between the mass fractions of the phases. ${ }^{5,6}$ As previously discussed, given that the structures of the different phases are not the same, this is not a valid assumption, and separate response factors must be taken into account. Mazzanti et al. ${ }^{7}$ introduced an ingenious way to experimentally derive response factors on the basis of solid fat content (SFC) determinations by low-resolution nuclear magnetic resonance (NMR). SFC determination has been used in fat crystallization studies to relate synchrotron SAXS patterns to $\alpha$ and $\beta^{\prime}$ mass fractions in palm oil ${ }^{7}$ and a blend of milk fat fractions. ${ }^{17}$ However, to our knowledge this approach has not been combined with wideangle XRD, a more accessible technique that also provides $\beta$ and $\beta^{\prime}$ differentiation.

Building on previous works, we propose a QPA method based on full XRD pattern decomposition combined with response factors derived from SFC measurements. The use of full XRD pattern decomposition renders precise identification and quantification of polymorphic transitions, even for complex systems with coexisting liquid, $\alpha, \beta^{\prime}$, and $\beta$ phases. With the use of individual response factors, experimentally derived from SFC measurements, this approach allows phase quantification without structural information or internal 
standards. Furthermore, through full-pattern decomposition, this method allows us to detect more subtle changes in the crystalline phases over time, manifested as peak broadenings and shifts, which can be related to changes in structure and composition. In the present work, we show how the proposed method can be used to quantify the evolving liquid and polymorphic phases of fats during crystallization, despite their complex composition and crystallization behavior. To illustrate the flexibility of the method, we selected two natural fats with contrasting origin and composition: palm oil and milk fat. To demonstrate how this method can be used to study systems displaying a complex crystallization behavior, we applied crystallization temperatures that resulted in high noncrystalline contributions and coexisting $\alpha$ and $\beta^{\prime}$ phases. To further challenge the robustness of the method, an additional crystallization temperature was used to study coexisting liquid, $\alpha, \beta^{\prime}$, and $\beta$ phases in milk fat.

\section{EXPERIMENTAL SECTION}

2.1. Materials. Anhydrous milk fat (AMF) was provided by FrieslandCampina (The Netherlands); the composition of this sample has been reported in detail elsewhere. ${ }^{22}$ Refined bleached and deodorized palm oil (PO) was provided by Sime Darby Unimills (The Netherlands). Both samples were stored at $-20{ }^{\circ} \mathrm{C}$ in glass containers and were melted at $45{ }^{\circ} \mathrm{C}$ prior to the analyses.

2.2. X-ray Diffraction Data Collection. To collect the diffraction patterns, a powder X-ray diffractometer (D8 Advance, Bruker) with a $\mathrm{Cu}$ anode $(\mathrm{K} \alpha \lambda 0.15406 \mathrm{~nm})$ was used, operated at $40.0 \mathrm{kV}$ and $40.0 \mathrm{~mA}$. To control the temperature, we used a lowtemperature chamber equipped with a $140 \mu \mathrm{L}$ sample holder (TTK600, Anton Paar). The molten samples were loaded into the sample holder and were kept at $65^{\circ} \mathrm{C}\left(\sim 29^{\circ} \mathrm{C}\right.$ above their melting points) for $10 \mathrm{~min}$ to erase crystal memory, and later cooled to 15 or $17^{\circ} \mathrm{C}$ using a cooling rate of $5{ }^{\circ} \mathrm{C} / \mathrm{min}$. The diffraction patterns were collected in Bragg-Brentano geometry over the wide-angle region from 15 to $30^{\circ} 2 \theta$, equivalent to a reciprocal lattice spacing $q\left(4 \pi \lambda^{-1}\right.$ $\sin \theta$ ) range from 10.6 to $21.1 \mathrm{~nm}^{-1}$. The patterns were recorded taking $0.1 \mathrm{~s}$ per $0.02^{\circ}$ increment, with a fixed divergence slit of $0.6 \mathrm{~mm}$ and using a detector opening of $2.914^{\circ}$. The scans were taken continuously every $96.5 \mathrm{~s}$ during the first $2 \mathrm{~h}$ of isothermal crystallization. To interpret the diffraction patterns, the scattering angle $2 \theta$ was converted according to Bragg's law to $d$ spacing.

2.3. Solid Fat Content Determination by NMR. The solid fat content (SFC) was measured during isothermal crystallization at 15 and $17{ }^{\circ} \mathrm{C}$ using a low-resolution nuclear magnetic resonance (NMR) spectrometer (Minispec mq20; Bruker). Approximately $2 \mathrm{~mL}$ of molten fat was placed in the NMR tubes and was kept at $65^{\circ} \mathrm{C}$ for $1 \mathrm{~h}$ to erase the crystal memory. Then, the sample was cooled at a rate of $5{ }^{\circ} \mathrm{C} / \mathrm{min}$ until the crystallization temperature $\left(15\right.$ or $\left.17^{\circ} \mathrm{C}\right)$ was reached. Using an external water bath, the measuring cell was kept at the crystallization temperature to ensure that the crystallization would take place isothermally during the measuring period.

2.4. Quantitative Phase Analysis. To achieve phase quantification, we followed three steps: (1) qualitative analysis of XRD patterns, (2) full pattern decomposition, and (3) derivation of response factors. All data management, visualization, and analysis described here were performed using the $\mathrm{R}$ programming language. ${ }^{23}$

2.4.1. Qualitative Analysis. The time-resolved XRD scans were visually inspected to identify the polymorphic phases present according to their characteristic diffraction peaks as described in the literature $^{3,4}$ (Table 1). On the basis of this qualitative analysis, the scans were classified depending on the polymorphs observed.

2.4.2. Decomposition of XRD Patterns. To quantify the contribution of the liquid and each polymorphic phase to the diffraction pattern, we fully decomposed the XRD scans into their constituent diffraction peaks. The diffraction patterns were decomposed by nonlinear least-squares fitting of a set of Pearson VII distribution functions per phase to the recorded intensity as a function
Table 1. Assignment of Polymorphs According to Their Characteristic $d$ Spacings $(\mathrm{nm})$ as Described in the Literature $^{3,4}$

\begin{tabular}{ccc}
$\alpha$ & $\beta^{\prime}$ & $\beta$ \\
$\sim 0.415$ & $\sim 0.420$ and $\sim 0.380$ or $\sim 0.427, \sim 0.397$ and $\sim 0.371$ & $\sim 0.46$ \\
\hline
\end{tabular}

of $q$. A Pearson VII function is defined by four parameters describing the width $w$, the height $I_{\max }$ the position $q_{0}$, and the shape $m$ as

$$
I(q)=I_{\max } \frac{w^{2 m}}{\left[w^{2}+\left(2^{1 / m}-1\right)\left(q-q_{0}\right)^{2}\right]^{m}}
$$

Due to the shape parameter $m$, the Pearson type VII function ranges from Cauchy to Gaussian profiles that render the versatility needed to describe the broad liquid curve and all of the diffraction peaks observed. The models fitted to the diffraction patterns were composed of a constant background and a Pearson VII function per peak. The number of functions and their approximated positions were selected for each polymorphic phase according to the peaks described in the literature ${ }^{3,4}$ (Table 1). The phases considered for the decomposition of a given pattern were those identified in the qualitative analysis.

First, a pattern with little or no crystalline contribution was used to characterize the background and the liquid peak $q_{0, \text { Liquid }}$ by fitting a $q$ independent constant and a Pearson VII function placed at $\sim 0.454$ $\mathrm{nm} d$ spacing, respectively. For this, the first scan recorded was selected, as it displayed the pattern with the highest liquid fat contribution. The constant background obtained from this fit was subtracted from all of the other patterns. The $q_{0, \text { Liquid }}$ value obtained was used to fix the position of the liquid peak in the models fitted to the following scans recorded during crystallization. Subsequently, all of the patterns containing only a crystalline $\alpha$ phase and liquid contribution were decomposed by fitting a peak placed at $\sim 0.415 \mathrm{~nm}$ $d$ spacing and the liquid curve with a fixed position. All of the patterns containing a crystalline contribution from only the $\beta^{\prime}$ polymorph were decomposed by fitting models with the fixed-position liquid curve and a set of four $\beta^{\prime}$ peaks: one placed at $\sim 0.38 \mathrm{~nm}$ and three in the $0.40-$ $0.43 \mathrm{~nm} d$ spacing region.

Once the liquid and the individual crystalline contributions were characterized, the next step was to decompose those scans displaying more than one polymorph. For scans displaying $\beta^{\prime}$ and $\beta$, the $\beta^{\prime}$ contribution was fitted with the four crystalline peaks observed in scans with $\beta^{\prime}$ alone, and the $\beta$ contribution was fitted with a peak at $\sim 0.46 \mathrm{~nm}$. To deal with the highly overlapped $\alpha$ and $\beta^{\prime}$ peaks recorded during the transition period, the parameters for the position $q_{0}$ and the shape $m$ were fixed as the average of the estimated values from the scans containing the individual crystalline phases. In the case of $\beta^{\prime}$, also the ratio between the heights $I_{\max }$ of the four peaks were fixed as the average ratios estimated from the scans with only the $\beta^{\prime}$ crystalline contribution. The script written in $\mathrm{R}$ programming language to decompose the patterns, including the models used, is provided as Supporting Information.

To evaluate the agreement between the experimental data and the fitted decomposition model, the homogeneity of the residuals along $q$ were visually inspected and the weighted profile $R$ factors $R_{\text {wp }}$ were calculated as

$$
R_{\mathrm{wp}}=\sqrt{\frac{\sum_{i} w_{i}\left(I_{i}^{\mathrm{calc}}-I_{i}^{\mathrm{obs}}\right)^{2}}{\sum_{i} w_{i}\left(I_{i}^{\mathrm{obs}}\right)^{2}}}
$$

with $I_{i}^{\text {calc }}$ and $I_{i}^{\text {obs }}$ being the calculated and observed intensities at a given position $q_{i j}$ and the weight $w_{i}=1 / I_{i}^{\text {obs }}$.

2.4.3. Derivation of Response Factors. To proceed with the QPA, the functions used to decompose the patterns were integrated over the $q$ range studied to obtain the contributing area of each peak to the diffraction pattern. The areas of all peaks associated with one phase were added, and the contributing areas of the $n$ crystalline phases to 


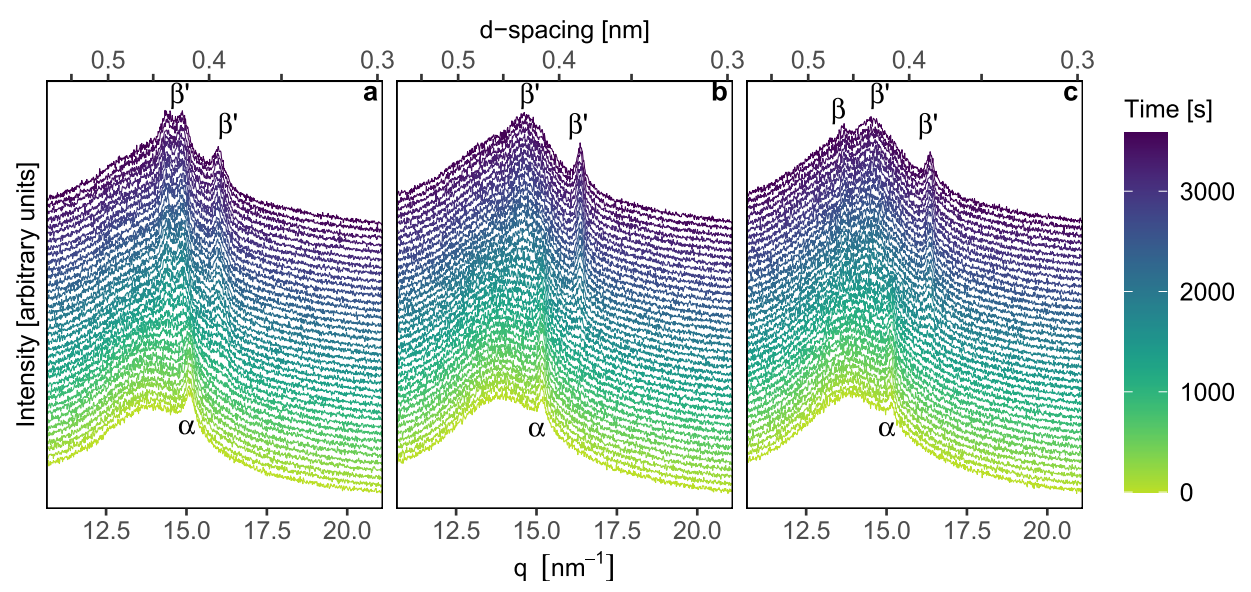

Figure 2. Time-resolved XRD patterns of (a) PO crystallizing at $15^{\circ} \mathrm{C}$, (b) AMF at $15^{\circ} \mathrm{C}$, and (c) AMF at $17^{\circ} \mathrm{C}$.

the diffraction pattern $A_{j}$ were related to their solid fat content contribution $\mathrm{SFC}_{j}$ as

$$
\operatorname{SFC}_{j}\left(A_{n}, \ldots A_{j}, A_{\mathrm{L}}\right)=\frac{A_{j} F_{j}}{A_{\mathrm{L}}+\sum_{j=1}^{n} A_{j} F_{j}}
$$

where $A_{\mathrm{L}}$ is the peak area of the liquid phase and $F_{j}$ is a response factor specific for each crystalline phase. This equation is an adaptation of the Hill and Howard relation for QPA using the Rietveld method, ${ }^{24}$ in which we have assigned a reference value of 1 to the liquid phase factor. Using a factor of 1 for the liquid phase normalizes the response factors of the crystalline phases and avoids the estimation of a redundant parameter. Assuming that the timeresolved solid fat content (SFC) is equal to the sum of the SFC fractions of the crystalline phases present at a given time $\mathrm{SFC}_{j}$, the phase-specific response factors $F_{j}$ can be estimated by nonlinear leastsquares optimization of substituting $\mathrm{SFC}_{j}$ with eq 3 .

$$
\operatorname{SFC}(\text { time })=\sum_{j=1}^{n} \operatorname{SFC}_{j}(\text { time })
$$

The uncertainty of the estimated response factors was assessed with the bootstrap method, resampling the mean centered residuals 1000 times. The bootstrapped estimates of the response factors were used to calculate the SFC fraction of each polymorph according to eq 3 . The standard errors of the bootstrapped estimates of the response factors were used in Monte Carlo simulations (10000) to predict the uncertainty in the phase quantifications. The $2.5-97.5 \%$ percentile was used to calculate the $95 \%$ confidence interval.

\section{RESULTS AND DISCUSSION}

To illustrate the proposed QPA approach, we selected natural fats with different composition: anhydrous milk fat (AMF) and palm oil (PO). In PO, two fatty acids (FAs), palmitic and oleic acid, make up more than $80 \%$ of the total fatty acids. ${ }^{25}$ As a consequence, $\sim 65 \%$ of the TAGs present in PO are composed of these two FAs only. ${ }^{26}$ In contrast, milk fat has a much more complex composition. A wide variety of TAG species compose milk fat, as a result of the presence of more than 400 FAs with different numbers, types and positions of unsaturation, and chain lengths that vary from 4 to 18 carbon atoms. ${ }^{27}$ As milk fat is one of the most complex natural fats, we selected AMF to demonstrate that this QPA method is also suitable for highly complex systems. Both samples were studied at $15{ }^{\circ} \mathrm{C}$, a temperature at which $\alpha$ to $\beta^{\prime}$ transitions can be observed within $1 \mathrm{~h}$. Additionally, AMF was studied at $17^{\circ} \mathrm{C}$ to illustrate the possibility to quantify coexisting $\alpha, \beta^{\prime}$, and $\beta$ phases.
As mentioned before, we follow three steps to quantify the crystalline and liquid phases. (i) We identify the different polymorphic phases present on the basis of the XRD pattern. (ii) We fully decompose all the recorded patterns to quantify the contribution of the liquid phase and each of the polymorphs. (iii) We derive phase-specific response factors to convert the contribution to the pattern of each polymorph to a measure of its mass fraction, the solid fat content.

3.1. Qualitative Analysis of XRD Patterns. The first step to quantify the phase evolution during crystallization is to classify the recorded patterns according to the phases present. This is the qualitative analysis commonly observed in fat crystallization studies using XRD. The time-resolved XRD scans were visually inspected to identify the polymorphs according to their characteristic diffraction peaks as described in the literature. ${ }^{3,4}$ The time-resolved XRD scans of the three experiments, $\mathrm{PO}$ at $15^{\circ} \mathrm{C}, \mathrm{AMF}$ at $15^{\circ} \mathrm{C}$, and $\mathrm{AMF}$ at $17^{\circ} \mathrm{C}$, are shown in Figure 2.

At $15{ }^{\circ} \mathrm{C}$, the XRD scans of PO (Figure 2a) evolved in a similar way as AMF (Figure 2b). In both cases, the first scans already show the characteristic $\alpha$ peak with a $d$ spacing of $\sim 0.415 \mathrm{~nm}$. After $\sim 700 \mathrm{~s}$ for PO and $\sim 1000 \mathrm{~s}$ for AMF at 15 ${ }^{\circ} \mathrm{C}$, the scans show a transition period in which the $\alpha$ contribution decreases and the distinctive $\beta^{\prime}$ peaks with $d$ spacings of $\sim 0.38 \mathrm{~nm}$ and in the $0.40-0.44 \mathrm{~nm}$ region appear. As the $\alpha$ contribution diminishes, only the $\beta^{\prime}$ contribution is observed in the scans recorded after this transition period, which remains until the end of $1 \mathrm{~h}$ of crystallization. A similar evolution was also observed for AMF at $17{ }^{\circ} \mathrm{C}$ (Figure 2c); however, at this temperature a characteristic $\beta$ peak with a $d$ spacing of $\sim 0.46 \mathrm{~nm}$ is also observed alongside the $\beta^{\prime}$ contribution. In all of the diffraction patterns, regardless of the sample or the temperature, the peaks of the different polymorphs overlap with a broad liquid peak with $d$ spacing $\sim 0.45 \mathrm{~nm}$.

3.2. XRD Pattern Decomposition. In the qualitative analysis, the XRD patterns were classified into three stages of the crystallization process: (1) the formation of $\alpha$ from the melt, (2) the transition from $\alpha$ to $\beta^{\prime}$ or from $\alpha$ to $\beta^{\prime}$ and $\beta$, and (3) the growth of $\beta^{\prime}$ and $\beta$ in the absence of $\alpha$. For the quantitative analysis, the classified XRD scans were fully decomposed to quantify the contribution of each phase identified in the qualitative analysis.

The XRD patterns were fully decomposed by fitting a model based on Pearson VII functions (eq 1). The first scan recorded 


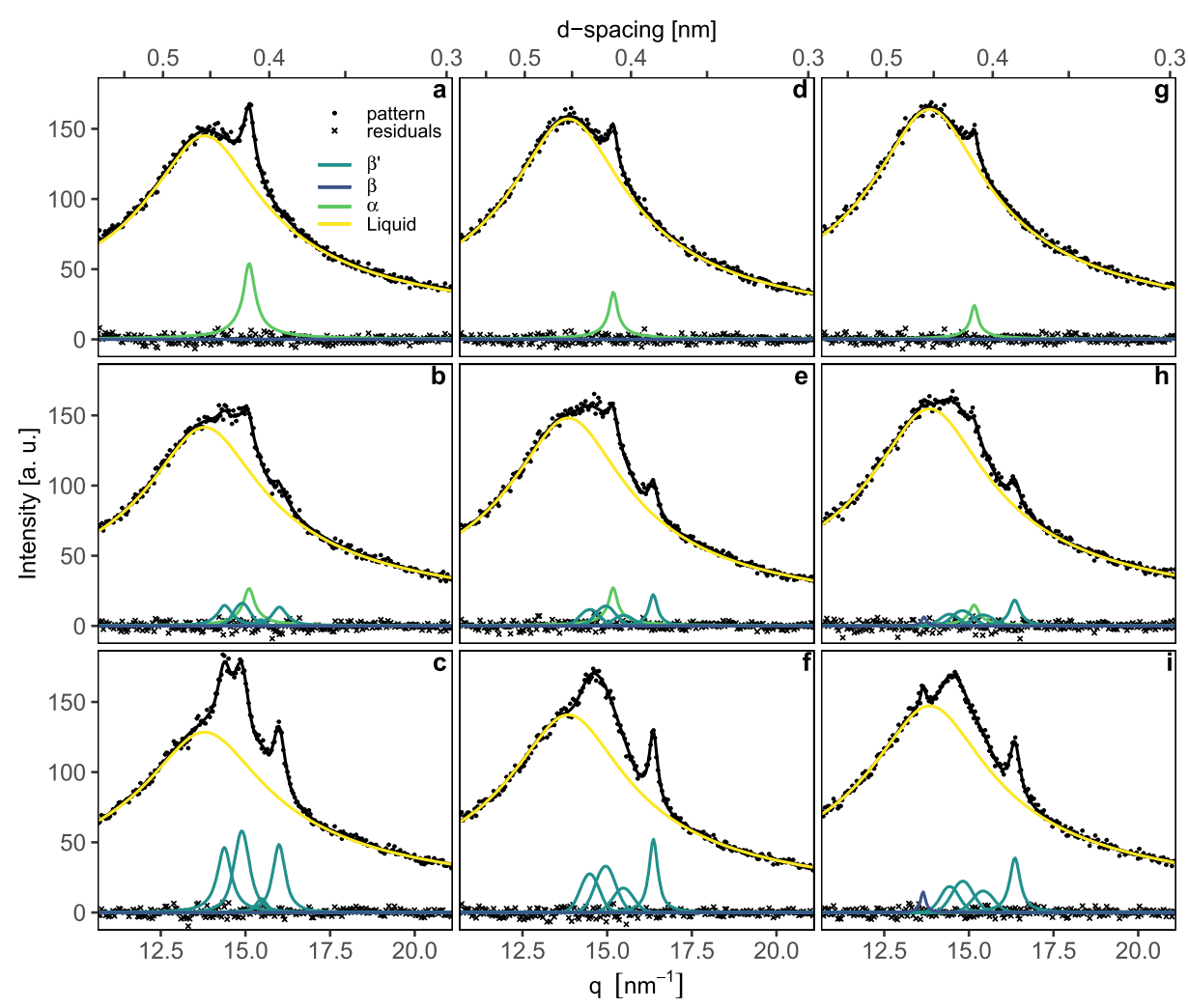

Figure 3. Decomposition of XRD patterns of $P O$ at $15{ }^{\circ} \mathrm{C}(\mathrm{a}-\mathrm{c}), \mathrm{AMF}$ at $15^{\circ} \mathrm{C}(\mathrm{d}-\mathrm{f})$ and $\mathrm{AMF}$ at $17{ }^{\circ} \mathrm{C}(\mathrm{g}-\mathrm{i})$. Plots (a), (d), and $(\mathrm{g})$ correspond to the first scans recorded, plots (b), (e), and (h) to scans from the polymorphic transition, and plots (c), (f), and (i) to the last scans recorded after $1 \mathrm{~h}$. The recorded intensities were fitted as a function of the reciprocal lattice spacing $q$ with sets of Pearson VII distribution functions.

in each experiment was decomposed with a model consisting of a constant background and two Pearson VII functions, one for the liquid contribution and one for the $\alpha$ contribution. The estimated constant background was removed in all of the remaining scans. Additionally, the position of the liquid peak extracted from the first scan was fixed in the remainder of the scans. The remaining background-subtracted patterns were thus decomposed with a model comprising this fixed-position liquid peak and one to four peaks for each crystalline phase. The crystalline phases included in the model used to decompose a given pattern were selected according to the qualitative analysis. A Pearson VII function was added to the model for each crystalline peak. The initial number and approximated position of the crystalline peaks were determined per polymorph on the basis of literature ${ }^{3,4}$ (Table 1 ). In the fitting, optimum values were estimated for the parameters defining the shape $m$, height $h$, width $w$, and position $q_{0}$ of the Pearson VII functions. A detailed description of the decomposition procedure is given in the Experimental Section.

Figure 3 shows representative examples of the pattern decomposition for each experiment and each stage of the crystallization process. The average estimated positions of the peaks of the three polymorphs and the liquid that best fit the diffraction patterns are provided in Table 2.

The diffraction pattern of the liquid phase was characterized in all the XRD patterns by a broad peak with $0.455 \pm 0.001 \mathrm{~nm}$ $d$ spacing. It is widely accepted that this broad XRD peak is characteristic of TAGs in the liquid state with conformationally disordered chains. ${ }^{28}$ As suggested by Wright et al., ${ }^{29}$ the presence of such a peak for molten TAGs may indicate lateral
Table 2. $d$ Spacing (nm) of the Peaks Found in Palm Oil and Anhydrous Milk Fat by Full Decomposition of their XRD Patterns

\begin{tabular}{lccc} 
& $\mathrm{PO}$ at $15{ }^{\circ} \mathrm{C}$ & $\mathrm{AMF}$ at $15^{\circ} \mathrm{C}$ & $\mathrm{AMF}$ at $17^{\circ} \mathrm{C}$ \\
\hline liquid & 0.456 & 0.454 & 0.454 \\
$\alpha$ & 0.416 & 0.414 & 0.415 \\
$\beta^{\prime}$ & 0.437 & 0.434 & 0.435 \\
& 0.422 & 0.42 & 0.424 \\
& 0.407 & 0.406 & 0.407 \\
$\beta$ & 0.393 & 0.384 & 0.384 \\
$\beta$ & & & 0.46 \\
\hline
\end{tabular}

packing in the liquid state; however, different models regarding its structural nature are still under debate. ${ }^{28,30-32}$

To characterize the contributions of the different polymorphs, we started with scans displaying a contribution of one polymorph only and later we moved on to decompose more complex patterns with coexisting crystalline phases. On the basis of the qualitative phase identification, patterns obtained at $15{ }^{\circ} \mathrm{C}$ at the beginning of the crystallization period were assumed to have only $\alpha$ and liquid contributions, where patterns at the end of the crystallization period were assumed to only have $\beta^{\prime}$ and liquid contributions. Once the $\alpha$ and $\beta^{\prime}$ phases were individually characterized, we calculated the average estimated values for the positions of their peaks. Additionally, we calculated the average ratios between the estimated heights of the main $\beta^{\prime}$ peak $(\sim 0.42 \mathrm{~nm} d$ spacing $)$ and the three other $\beta^{\prime}$ peaks. These average peak positions and ratios were used to decompose the patterns with coexisting crystalline phases recorded during the polymorphic transitions. 


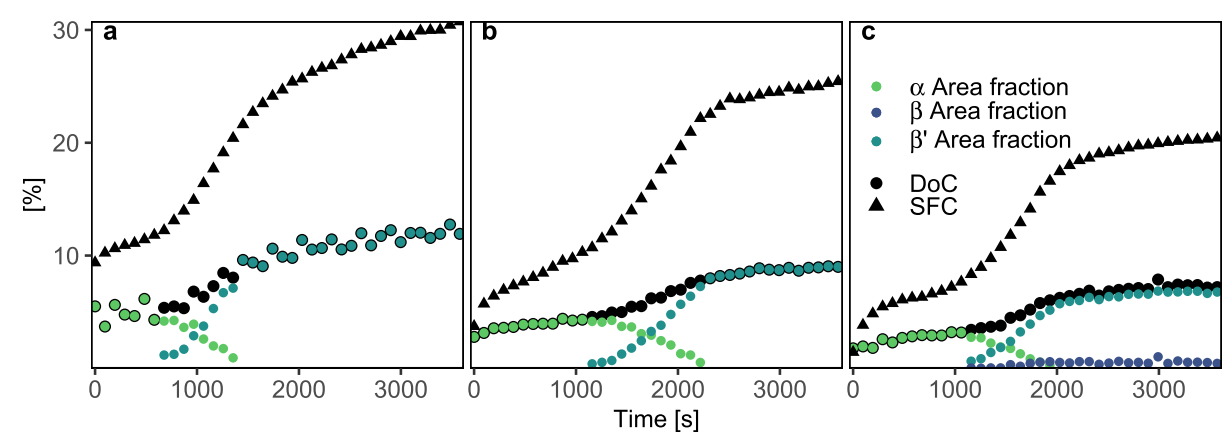

Figure 4. Total integrated intensity (area) contributed to the overall XRD pattern by each phase for (a) PO at $15^{\circ} \mathrm{C}$ and $\mathrm{AMF}(\mathrm{b})$ at $15{ }^{\circ} \mathrm{C}$ and (c) at $17{ }^{\circ} \mathrm{C}$. The degree of crystallinity (DoC) and the solid fat content (SFC) by NMR are included as measures of the crystalline fraction.

The patterns from the first stage of the crystallization processes, displaying $\alpha$ and liquid contribution only, were decomposed by fitting the broad liquid peak with $0.455 \pm$ $0.001 \mathrm{~nm} d$ spacing and an $\alpha$ peak with $0.415 \pm 0.001 \mathrm{~nm} d$ spacing. The results are shown in Figure $3 \mathrm{a}, \mathrm{d}, \mathrm{g}$, which correspond to the decomposition of the first scans for $\mathrm{PO}$ at $15{ }^{\circ} \mathrm{C}$, AMF at $15{ }^{\circ} \mathrm{C}$, and AMF at $17{ }^{\circ} \mathrm{C}$, respectively.

Figure $3 \mathrm{c}, \mathrm{f}$ show the scans after $1 \mathrm{~h}$ of crystallization at 15 ${ }^{\circ} \mathrm{C}$ for PO and AMF, respectively. In these scans, as well as in all other scans after the polymorphic transition at $15{ }^{\circ} \mathrm{C}$, the $\alpha$ contribution has disappeared and only the liquid and the $\beta^{\prime}$ peaks are visible. These patterns were decomposed fitting four $\beta^{\prime}$ peaks and the fixed-position liquid peak. The $\beta^{\prime}$ polymorph of PO is commonly characterized by only three diffraction peaks at $0.437,0.423$, and $0.39 \mathrm{~nm} .{ }^{33}$ However, decomposition of our PO scans using only those three $\beta^{\prime}$ peaks resulted in consistent underfitting in the $0.4 \mathrm{~nm} d$ spacing region, which could only be overcome by including a fourth smaller peak with $0.407 \mathrm{~nm} d$ spacing. Similarly, to properly fit the AMF $\beta^{\prime}$ patterns (Figure $3 \mathrm{f}$ ), four peaks with $d$ spacings of $0.434,0.42$, 0.406 , and $0.384 \mathrm{~nm}$ were needed, while only two peaks with $d$ spacings of $\sim 0.42$ and $\sim 0.38 \mathrm{~nm}$ are commonly mentioned for milk fat $\beta^{\prime}$ phases. ${ }^{5,16,34}$

Despite this apparent discrepancy with the data commonly reported for the $\beta^{\prime}$ peaks in PO and milk fat, no obvious difference can be found by visually comparing the shape of our XRD patterns and other reported patterns. ${ }^{5,33,34}$ The different numbers and positions of $\beta^{\prime}$ peaks reported in those studies and in ours might arise from different crystallization conditions or by the way the data were processed. When diffraction patterns are visually inspected, only the peaks with the highest intensities can be identified easily. As peaks often overlap with adjacent ones, their position can appear shifted and the presence of smaller peaks may be completely masked by larger peaks. This may lead to the identification of fewer peaks, whereas the pattern actually contains other smaller peaks. The presence of four separate $\beta^{\prime}$ peaks only became clear through pattern decomposition which, it should be noted, is not a common practice for the evaluation of complex fats. The presence of these four $\beta^{\prime}$ diffraction peaks is plausible, as Chapman $^{3}$ mentioned in his classification that the $\beta^{\prime}$ polymorph occasionally displays more peaks together with the 0.420 and $0.380 \mathrm{~nm} d$ spacing peaks. Additionally, both the occurrence of the four peaks and their corresponding $d$ spacings (Table 2) agree with Hoerr's description of $\beta^{\prime}$ polymorphs for commercial fats. ${ }^{35}$ On the basis of numerous observations for commercial fats, Hoerr concluded that the $\beta^{\prime}$ form always displays four diffraction peaks with spacings of
$0.435,0.420,0.403$, and $0.380 \mathrm{~nm} .^{35}$ Despite the reassuring agreement between the $\beta^{\prime}$ characteristics reported here with Hoerr's findings, it is worth mentioning that the $d$ spacings reported in Table 2 arise from a "peak by peak" fit without any structure-related constraints. Further interpretation of the $d$ spacings would require the inclusion of structural information in the analysis, which, as we discussed earlier, is not compatible with the complex composition of most fats. Nevertheless, the objective of our pattern decomposition is not to obtain the exact position of the peaks of a given polymorph but to quantify its area contribution to the pattern and, for this, our approach is sufficient.

Once the $\alpha$ and the $\beta^{\prime}$ contributions to the pattern were characterized individually, the $\beta$ contribution was characterized. For this, we decomposed the scans recorded after the polymorphic transition for AMF at $17{ }^{\circ} \mathrm{C}$ (Figure 3i). These scans showed both $\beta^{\prime}$ and $\beta$ contributions, but no $\alpha$ contribution. The $\beta$ diffraction pattern of TAGs is typically characterized by an intense peak with a $d$ spacing of $\sim 0.46 \mathrm{~nm}$ and two additional smaller peaks with spacings of $\sim 0.385$ and $\sim 0.365 \mathrm{~nm} .{ }^{35}$ In our patterns, the contribution to the pattern from the main $\beta$ peak was modeled with a Pearson VII function centered at $0.46 \mathrm{~nm}$; however, possible contribution from smaller $\beta$ peaks could not be distinguished from the 0.384 $\mathrm{nm} \beta^{\prime}$ peak. As the $0.384 \mathrm{~nm}$ peak recorded at $17{ }^{\circ} \mathrm{C}$ was $17 \%$ wider than the $\beta^{\prime}$ peak recorded in the absence of $\beta$ at $15{ }^{\circ} \mathrm{C}$, this indicates that the crystalline signal in the $0.36-0.38 \mathrm{~nm}$ region at $17{ }^{\circ} \mathrm{C}$ might be constructed from both $\beta^{\prime}$ and $\beta$ contributions. The $\beta$ contribution might thus not be determined by just the $0.46 \mathrm{~nm}$ peak but may have additional peaks in the $0.38 \mathrm{~nm}$ region. Therefore, the $0.46 \mathrm{~nm}$ peak might not represent the total $\beta$ fraction, leading to a slight underestimation of the $\beta$ contribution and overestimation of the $\beta^{\prime}$ contribution.

After characterizing the individual contributions of $\alpha, \beta^{\prime}$, and $\beta$ to the patterns recorded before and after the polymorphic transition, we used the values estimated for those patterns as a reference to decompose the transition patterns, in which $\alpha$ coexisted with $\beta^{\prime}$ and $\beta$. The average estimated position $q_{0}$ and shape $m$ of all the crystalline peaks obtained before were used as fixed parameters in the models applied to the scans from the polymorphic transition. Additionally, to model the $\beta^{\prime}$ contribution during the transition period, the ratio between the heights $I_{\max }$ of the four peaks were fixed as the average ratios estimated from the scans recorded after the transition. This way, only two parameters per polymorph were used as fitting parameters: $I_{\max }$ and $w$. The pattern decompositions of representative scans from the polymorphic transitions are 
shown in Figure $3 \mathrm{~b}, \mathrm{e}, \mathrm{h}$ for $\mathrm{PO}$ at $15^{\circ} \mathrm{C}$, AMF at $15{ }^{\circ} \mathrm{C}$, and AMF at $17^{\circ} \mathrm{C}$, respectively.

As can be seen in Figure 3, the fitted models based on Pearson VII functions adequately matched the experimental patterns. The good quality of the fit was judged on the basis of the residuals that were, in all cases, homogeneously distributed along q. Additionally, the good quality of each fit was confirmed by the reliability factor $R_{\mathrm{wp}}$, calculated according to eq $2 . R_{\text {wp }}$ values between 2 and $10 \%$ indicate a good-quality fit; ${ }^{36}$ in all of our decompositions, only values between $2.0 \%$ and $2.4 \%$ were obtained.

3.3. Derivation of Response Factors. After all of the time-resolved XRD patterns were decomposed, all of the obtained functions were integrated over the $q$ range studied to calculate the areas of the peaks. To obtain the total area contribution of $\beta^{\prime}$ to the pattern, the areas of the four separate $\beta^{\prime}$ peaks were summed. With this approach, we were able to calculate the area contribution to the pattern of each polymorphic phase as a function of time, even for those patterns displaying a dominant liquid contribution coexisting with three polymorphic phases. In Figure 4, the contribution of each polymorphic phase normalized by the total area is plotted as a function of time.

Besides calculating the contribution of the individual polymorphs to the diffraction pattern, we calculated the total contribution of the crystalline phases, the degree of crystallinity (DoC). The DoC is calculated as the fraction of the crystalline area over the total area of the pattern. ${ }^{37}$ The DoC is a common method to assess the ratio between crystalline and noncrystalline materials and has been used earlier in fat studies ${ }^{19,38}$ as a measure of the crystalline fraction.

Another measure of the crystalline fraction in fats is the SFC measured by NMR. The SFC is calculated by measuring the percentage of protons present in the solid state. As proton relaxation times for the liquid and solid state are different, this can be used to quantify the ratio between liquid and solid fat contributions. ${ }^{39}$ Although there tends to be a small difference of $0.5-1 \%$ between SFC and the actual solid mass fraction, ${ }^{39}$ SFC is commonly used as a measure of the solid mass fraction in fat studies. In Figure 4, these two measures of the crystalline fraction, the DoC (black circles) and the SFC (black triangles), can be compared. The large differences between DoC and SFC values indicate that the DoC based on XRD patterns alone is not a good measure for the crystalline mass fraction of fats. This difference is unsurprising, as XRD is sensitive to longrange order whereas NMR is sensitive to both short- and longrange order. Consequently, NMR will record a greater SFC in comparison to DoC. Lopez-Rubio et al. ${ }^{40}$ used a similar approach to evaluate single- and double-helical order in starch. On the basis of our results, it is clear that specific response factors are needed to relate the diffraction patterns to mass fractions.

These specific response factors are needed for each phase separately. As we have already discussed, the fact that each phase has a specific response factor arises from its crystallographic properties (i.e., atomic arrangement within the unit cell). The phase-specific response factors were estimated by nonlinear least-squares analysis according to eqs 3 and 4, using the integrated decomposed XRD patterns and, as a measure of the total crystalline mass, time-resolved SFC. To evaluate the uncertainty of the estimation, we bootstrapped the mean centered residuals, creating 1000 resampled data sets from which the response factors were estimated again. The bootstrap estimates of the response factors and their standard errors are included in Table 3.

Table 3. Response Factors Empirically Derived to Convert the Area Contributing to the XRD Pattern by Each Phase to Its SFC Fraction ${ }^{a}$

$\begin{array}{cccc} & \mathrm{PO} \text { at } 15{ }^{\circ} \mathrm{C} & \mathrm{AMF} \text { at } 15^{\circ} \mathrm{C} & \mathrm{AMF} \text { at } 17^{\circ} \mathrm{C} \\ F_{\alpha} & 2.29(0.1) & 2.29(0.05) & 2.35(0.07) \\ F_{\beta^{\prime}} & 3.02(0.04) & 3.42(0.03) & 3.35(0.06) \\ F_{\beta^{\prime}+\beta} & & 3.2(0.03) \\ F_{\beta} & & 1.43(0.63) \\ { }^{a} \text { The standard errors are included in parentheses. } & \end{array}$

In a Rietveld analysis, the response factors reflect the volume of the unit cell and the mass of its contents. To derive this structural information from a Rietveld analysis, the so-called "fundamental parameters approach" is followed, which requires the use of rational physical quantities to model the diffraction pattern. ${ }^{12}$ In our case, an empirical approach is followed to model the pattern and no attempt is made to associate the parameters with physical quantities. Although the response factors derived here do not have physical significance, they provide a fast and easy way to relate contributions to the pattern to mass fraction.

The obtained response factors and their standard errors are presented in Table 3. As expected, the response factors obtained differ per crystalline phase. This is in agreement with the differences reported in SAXS studies between $\alpha$ and $\beta^{\prime}$ phases for palm oil ${ }^{7}$ and a blend of milk fat fractions. ${ }^{17}$ For the experiments at $15{ }^{\circ} \mathrm{C}$, in which only two crystalline phases coexisted, $\alpha$ and $\beta^{\prime}$, we estimated their response factors with high precision, as is reflected in the small standard errors. However, when $\alpha, \beta^{\prime}$, and $\beta$ coexisted, as in the crystallization of $\mathrm{AMF}$ at $17{ }^{\circ} \mathrm{C}$, the precise determination of individual response factors was more complicated. For AMF at $17{ }^{\circ} \mathrm{C}$, we observed a high correlation between the contributions of $\beta^{\prime}$ and $\beta$ to the pattern. As can be seen in Figure $4 \mathrm{c}, \beta$ appeared only $\sim 200 \mathrm{~s}$ later than $\beta^{\prime}$, and the contributions to the pattern of both phases increased mainly during the transition period. Due to this high correlation between $\beta^{\prime}$ and $\beta$ contributions to the pattern, the uncertainty in the determination of the individual response factors is high. Given this high correlation, we followed a different procedure to allow a more precise estimation of the different factors. First, the response factor for the $\alpha$ phase, $F_{\alpha}$, was determined simultaneously with a response factor for $\beta^{\prime}$ and $\beta$ combined, $F_{\beta^{\prime}+\beta}$. $F_{\beta^{\prime}+\beta}$, also presented in Table 3, was used to calculate the added SFC of $\beta^{\prime}$ and $\beta\left(\mathrm{SFC}_{\beta^{\prime}+\beta}\right)$ on the basis of their combined contribution to the pattern $\left(A_{\beta^{\prime}}+A_{\beta}\right)$. These $\operatorname{SFC}_{\beta^{\prime}+\beta}$ values and the individual contributions of $\beta^{\prime}$ and $\beta$ to the pattern $\left(A_{\beta^{\prime}}\right.$ and $\left.A_{\beta}\right)$ were then used as the input variables for eqs 3 and 4 to obtain the individual response factors $F_{\beta^{\prime}}$ and $F_{\beta}$. Finally, $F_{\beta^{\prime}}$ and $F_{\beta}$ were used to calculate the individual SFC values of $\beta^{\prime}$ and $\beta$. We should note that, due to the colinear relationship of the $\beta^{\prime}$ and $\beta$ contributions to the total area of the pattern, their estimated response factors were also correlated. The high correlation between $F_{\beta^{\prime}}$ and $F_{\beta}$, together with the relatively small $\beta$ contribution to the pattern, increased the uncertainty in the estimation of $F_{\beta}$, as is reflected in its associated relatively high standard error (Table 3).

The phase-specific response factors (Table 3) were used to convert the individual contribution of each phase to the total 


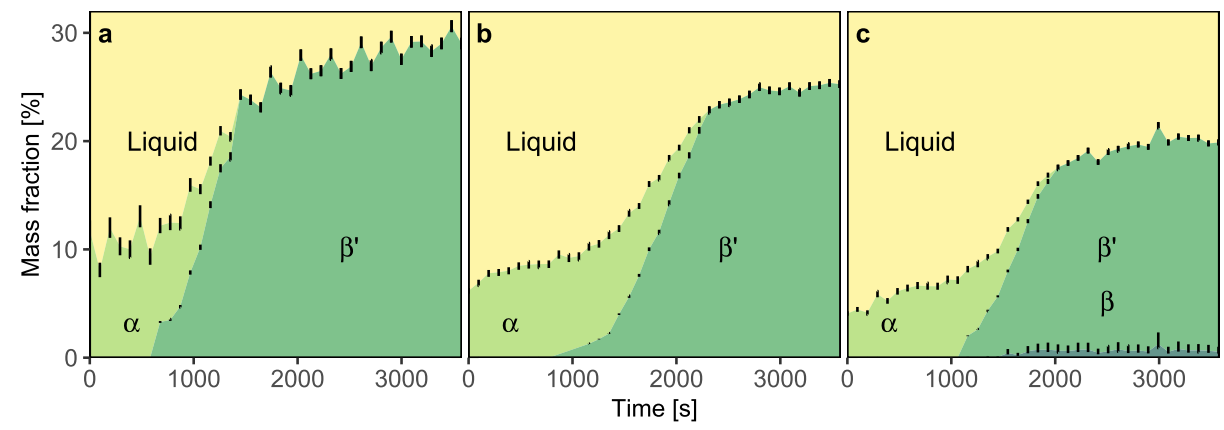

Figure 5. Phase transition diagram for (a) PO at $15{ }^{\circ} \mathrm{C}$ and $\mathrm{AMF}$ (b) at $15{ }^{\circ} \mathrm{C}$ and (c) at $17^{\circ} \mathrm{C}$. The error bars correspond to the $95 \%$ confidence interval.

area of the XRD pattern (Figure 4) into its corresponding contributions to the total SFC according to eq 3 . This allowed us to calculate the mass contribution of each polymorphic phase over time and construct phase transition diagrams. Parts $\mathrm{a}-\mathrm{c}$ of Figure 5 display the phase transition diagrams for $\mathrm{PO}$ at $15{ }^{\circ} \mathrm{C}$, AMF at $15{ }^{\circ} \mathrm{C}$, and $\mathrm{AMF}$ at $17{ }^{\circ} \mathrm{C}$, respectively, showing the evolution of the liquid fat content and the SFC fraction of each polymorphic phase during crystallization.

As can be seen in Figure 4a, PO crystallized rapidly into $\alpha$, which already contributed to an SFC of $12 \%$ by the time the first XRD scan was recorded. This $\alpha$ content remained constant until the first $\beta^{\prime}$ pattern was recorded after $677 \mathrm{~s}$. Within the following $774 \mathrm{~s}$, the amount of $\alpha$ decreased until its characteristic diffraction peak could no longer be detected, while the amount of the $\beta^{\prime}$ phase rapidly increased, reaching $24 \%$. This $\alpha$ to $\beta^{\prime}$ polymorphic transition lasted until $1451 \mathrm{~s}$, after which the amount of $\beta^{\prime}$ kept increasing at a rate much slower than that during the polymorphic transition period. After $1 \mathrm{~h}$ of crystallization, the total SFC reached a value of $31 \%$.

In the phase transition diagram of AMF at $15^{\circ} \mathrm{C}$ (Figure $5 b)$, we see a phase evolution similar to that for PO. In the first stage, the amount of $\alpha$ increased from an initial 6\% to $10 \%$ within 869 s, after which it remained constant. During the polymorphic transition, which took place between 1158 and 2316 s, the $\alpha$ content started to decrease until it could no longer be detected. At the same time, the $\beta^{\prime}$ phase appeared and rapidly increased, reaching an SFC of $23 \%$. After the polymorphic transition, a very limited amount of $\beta^{\prime}$ was then still formed, as the SFC increased only by an additional $3 \%$.

The phase transition diagram of AMF at $17^{\circ} \mathrm{C}$ (Figure 5c) shows the effect of a different crystallization temperature. Due to the higher temperature, the crystallization process is slower than that at $15{ }^{\circ} \mathrm{C}$. During the initial stage, TAGs crystallized in the $\alpha$ form and contributed to 4\% SFC. The amount of $\alpha$ crystals increased to its maximum (7\% SFC) after $965 \mathrm{~s}$ and remained constant until $1158 \mathrm{~s}$, when the polymorphic transition started. In comparison to $15{ }^{\circ} \mathrm{C}$, at which only $\beta^{\prime}$ was formed, both $\beta^{\prime}$ and $\beta$ phases appeared at this higher temperature of $17{ }^{\circ} \mathrm{C}$. The polymorphic transition at $17^{\circ} \mathrm{C}$ seems to start around the same time as for $15{ }^{\circ} \mathrm{C}(1158 \mathrm{~s})$; however, at $17{ }^{\circ} \mathrm{C}$, the $\alpha$ content decreased to $0 \%$ already $289 \mathrm{~s}$ earlier than that at $15{ }^{\circ} \mathrm{C}$ (after $2027 \mathrm{~s}$ instead of $2316 \mathrm{~s}$ ). During the transition period, the amount of $\beta^{\prime}$ and $\beta$ increased steeply from 0 to $18 \%$ and it only increased $2 \%$ more in the remaining time of the $1 \mathrm{~h}$ studied.

The amount of $\beta$ formed at $17{ }^{\circ} \mathrm{C}$ within the transition period was calculated to be $1 \%$ and remained constant until the end. As we discussed earlier, it is possible that the area contribution to the pattern of $\beta$ was underestimated, leading to underestimation and overestimation of $\beta$ and $\beta^{\prime}$ mass fractions, respectively. Furthermore, the relatively large error bars for the $\beta$ phase in Figure $5 \mathrm{c}$ show that the precision in the quantitative analysis may be limited in this specific case. As we have already discussed, the higher uncertainty in the determination of the $\beta$ fraction was the result of its relatively small contribution to the pattern and the high correlation between the evolution of $\beta^{\prime}$ and $\beta$. Even though in this case there is a slight underestimation of the $\beta$ fraction in AMF, this simple approach is able to determine the different mass fractions in complicated mixtures. Further characterization of $\beta$ diffraction patterns in milk fat could help to solve this problem for future studies.

The phase transition diagrams presented in Figure 5 demonstrate how the proposed approach allows the quantification of coexisting noncrystalline and different crystalline phases evolving during early crystallization stages, even for fats with a complex composition. Until now, the phase quantification of such fats in terms of a reasonable approximation of the mass fractions (SFC) has only been done using synchrotron SAXS. ${ }^{7,17}$ The approach presented here relies on XRD in the wide-angle region, which is a more accessible technique. This makes phase quantification for complex fats possible with simple X-ray diffractometers. A second advantage arises from the direct use of XRD patterns in the wide-angle region to quantify different polymorphs, rather than through their associated long spacings using SAXS. We have even quantified coexisting liquid, $\alpha, \beta^{\prime}$, and $\beta$ phases, which would have not been possible using only SAXS patterns.

\section{CONCLUSION}

Typical phase quantification methods for multicomponent materials are not used to study common fats, given their highly complex composition and crystallization behavior. The aim of this paper was to present a new quantitative phase analysis (QPA) method that is able to characterize and quantify the evolution of different polymorphic phases in the early crystallization of complex fats. We discussed the versatility of the method to characterize the crystallization of complex fats, by producing quantitative phase transition diagrams for both palm oil and anhydrous milk fat. This was achieved without the need for structural information on the different phases and using diffraction patterns obtained by XRD in the wide-angle region.

The method introduced here is based on the full decomposition of XRD patterns, combined with the estimation of phase-specific response factors from solid fat content (SFC) 
measurements. The importance of the phase-specific response factors was shown by comparing the DoC and the SFC values. From this comparison, we also concluded that the DoC does not provide a good estimate for the ratio between the mass of the solid fraction and the molten TAGs in fats. In contrast with other QPA methods applied to fats, the method proposed here has the advantage of (i) making QPA accessible even with inhouse X-ray diffraction systems that cannot reach small scattering angles and (ii) allowing quantification of coexisting $\beta^{\prime}$ and $\beta$ phases that typically display only one SAXS peak. The phase transition diagrams obtained through this QPA method will provide reliable and detailed quantitative input for kinetic studies that will prove useful in expanding our understanding of the mechanisms involved in fat crystallization.

\section{ASSOCIATED CONTENT}

\section{s) Supporting Information}

The Supporting Information is available free of charge at https://pubs.acs.org/doi/10.1021/acs.cgd.0c00416.

$\mathrm{R}$ programming language script used to decompose the time-resolved XRD patterns of AMF and PO (PDF)

\section{AUTHOR INFORMATION}

\section{Corresponding Author}

Elke Scholten - Physics and Physical Chemistry of Foods, Wageningen University, Wageningen, The Netherlands; Phone: +31 (0)317 482288; Email: elke.scholten@wur.nl

\section{Authors}

Naomi Arita-Merino - Physics and Physical Chemistry of Foods and Dairy Science and Technology, Food Quality and Design, Wageningen University, Wageningen, The Netherlands; (1) orcid.org/0000-0002-5293-5751

Hein van Valenberg - Dairy Science and Technology, Food Quality and Design, Wageningen University, Wageningen, The Netherlands

Elliot Paul Gilbert - Australian Centre for Neutron Scattering, Australian Nuclear Science and Technology Organisation,

Kirrawee, New South Wales 2232, Australia

Complete contact information is available at:

https://pubs.acs.org/10.1021/acs.cgd.0c00416

\section{Notes}

The authors declare no competing financial interest.

\section{ACKNOWLEDGMENTS}

We gratefully acknowledge the financial support from CONACYT (grant number 474601). We thank FrieslandCampina for providing access to the AMF sample and the NMR equipment, especially to Antoinette Toebes and Gerund van Binsbergen for their technical support.

\section{REFERENCES}

(1) Wesdorp, L. H.; van Meeteren, J. A.; de Jong, S.; van der Giessen, R.; Overbosch, P.; Grootscholten, P. A. M.; Struik, M.; Royers, E.; Don, A.; de Loos, T.; Peters, C.; Gandasasmita, I. In Structure and properties of fat crystal networks, 2nd ed.; CRC Press: Boca Raton, FL, 2012; pp 241-418.

(2) D’Souza, V.; DeMan, J. M.; DeMan, L. Short spacings and polymorphic forms of natural and commercial solid fats: A review. $J$. Am. Oil Chem. Soc. 1990, 67, 835-843.

(3) Chapman, D. The Polymorphism of Glycerides. Chem. Rev. 1962, 62, 433-456.
(4) Larsson, K.; Cyvin, S. J.; Rymo, L.; Bowie, J. H.; Williams, D. H.; Bunnenberg, E.; Djerassi, C.; Records, R. Classification of Glyceride Crystal Forms. Acta Chem. Scand. 1966, 20, 2255-2260.

(5) Lopez, C.; Lesieur, P.; Bourgaux, C.; Keller, G.; Ollivon, M. Thermal and Structural Behavior of Milk Fat. J. Colloid Interface Sci. 2001, 240, 150-161.

(6) Dewettinck, K.; Foubert, I.; Basiura, M.; Goderis, B. Phase Behavior of Cocoa Butter in a Two-Step Isothermal Crystallization. Cryst. Growth Des. 2004, 4, 1295-1302.

(7) Mazzanti, G.; Marangoni, A. G.; Idziak, S. H. J. Modeling phase transitions during the crystallization of a multicomponent fat under shear. Phys. Rev. E 2005, 71, 041607.

(8) Timms, R. Phase behaviour of fats and their mixtures. Prog. Lipid Res. 1984, 23, 1-38.

(9) Gresti, J.; Bugaut, M.; Maniongui, C.; Bezard, J. Composition of Molecular Species of Triacylglycerols in Bovine Milk Fat. J. Dairy Sci. 1993, 76, 1850-1869.

(10) Lopez, C. In Crystallization of lipids: Fundamentals and applications in food, cosmetics, and pharmaceuticals, 1st ed.; Sato, K., Ed.; Wiley: Hoboken, NJ, 2018; pp 283-321.

(11) Lopez, C.; Lesieur, P.; Bourgaux, C.; Ollivon, M. Thermal and Structural Behavior of Anhydrous Milk Fat. 3. Influence of Cooling Rate. J. Dairy Sci. 2005, 88, 511-526.

(12) Pecharsky, V. K.; Zavalij, P. Y. In Fundamentals of powder diffraction and structural characterization of materials, 2nd ed.; Springer US: Boston, MA, 2009; pp 151-202.

(13) Hill, R. J. Expanded use of the Rietveld method in studies of phase abundance in multiphase mixtures. Powder Diffr. 1991, 6, 7477.

(14) Kalnin, D.; Lesieur, P.; Artzner, F.; Keller, G.; Ollivon, M. Systematic investigation of lard polymorphism using combined DSC and time-resolved synchrotron X-ray diffraction. Eur. J. Lipid Sci. Technol. 2005, 107, 594-606.

(15) Bugeat, S.; Briard-Bion, V.; Pérez, J.; Pradel, P.; Martin, B.; Lesieur, S.; Bourgaux, C.; Ollivon, M.; Lopez, C. Enrichment in unsaturated fatty acids and emulsion droplet size affect the crystallization behaviour of milk triacylglycerols upon storage at $4^{\circ} \mathrm{C}$. Food Res. Int. 2011, 44, 1314-1330.

(16) Mazzanti, G.; Guthrie, S. E.; Sirota, E. B.; Marangoni, A. G.; Idziak, S. H. J. Effect of Minor Components and Temperature Profiles on Polymorphism in Milk Fat. Cryst. Growth Des. 2004, 4, 13031309.

(17) Cisneros, A.; Mazzanti, G.; Campos, R.; Marangoni, A. G. Polymorphic Transformation in Mixtures of High- and Low-Melting Fractions of Milk Fat. J. Agric. Food Chem. 2006, 54, 6030-6033.

(18) Scarlett, N. V. Y.; Madsen, I. C. Quantification of phases with partial or no known crystal structures. Powder Diffr. 2006, 21, 278284.

(19) Calligaris, G. A.; da Silva, T. L.T.; Ribeiro, A. P. B.; dos Santos, A. O.; Cardoso, L. P. On the quantitative phase analysis and amorphous content of triacylglycerols materials by X-ray Rietveld method. Chem. Phys. Lipids 2018, 212, 51-60.

(20) van Mechelen, J. B.; Peschar, R.; Schenk, H. Structures of mono-unsaturated triacylglycerols. IV. The highest melting $\beta^{\prime}-2$ polymorphs of trans-mono-unsaturated triacylglycerols and related saturated TAGs and their polymorphic stability. Acta Crystallogr., Sect. B: Struct. Sci. 2008, 64, 249-259.

(21) van Langevelde, A.; Peschar, R.; Schenk, H. Structure of $\beta$ trimyristin and $\beta$-tristearin from high-resolution $\mathrm{X}$-ray powder diffraction data. Acta Crystallogr., Sect. B: Struct. Sci. 2001, 57, 372377.

(22) Yener, S.; van Valenberg, H. J.F. Characterisation of triacylglycerols from bovine milk fat fractions with MALDI-TOFMS fragmentation. Talanta 2019, 204, 533-541.

(23) R Core Team: R: A language and environment for statistical computing; R Foundation for Statistical Computing: Vienna, Austria, 2019. 
(24) Hill, R. J.; Howard, C. J. Quantitative phase analysis from neutron powder diffraction data using the Rietveld method. J. Appl. Crystallogr. 1987, 20, 467-474.

(25) Alimentarius, C. Codex standard for named vegetable oils. Codex stan 1999, 210, 1-13.

(26) Braipson-Danthine, S.; Gibon, V. Comparative analysis of triacylglycerol composition, melting properties and polymorphic behavior of palm oil and fractions. Eur. J. Lipid Sci. Technol. 2007, 109, 359-372.

(27) Jensen, R. G. The Composition of Bovine Milk Lipids: January 1995 to December 2000. J. Dairy Sci. 2002, 85, 295-350.

(28) Corkery, R. W.; Rousseau, D.; Smith, P.; Pink, D. A.; Hanna, C. B. A Case for Discotic Liquid Crystals in Molten Triglycerides. Langmuir 2007, 23, 7241-7246.

(29) Wright, A. J.; McGauley, S. E.; Narine, S. S.; Willis, W. M.; Lencki, R. W.; Marangoni, A. G. Solvent Effects on the Crystallization Behavior of Milk Fat Fractions. J. Agric. Food Chem. 2000, 48, 10331040.

(30) Cebula, D. J.; McClements, D. J.; Povey, M. J. W.; Smith, P. R. Neutron diffraction studies of liquid and crystalline trilaurin. J. Am. Oil Chem. Soc. 1992, 69, 130-136.

(31) Larsson, K. Molecular Arrangement in Glycerides. Fette, Seifen, Anstrichm. 1972, 74, 136-142.

(32) Sadeghpour, A.; Parada, M. L.; Vieira, J.; Povey, M.; Rappolt, M. Global Small-Angle X-ray Scattering Data Analysis of Triacylglycerols in the Molten State (Part I). J. Phys. Chem. B 2018, 122, 10320-10329.

(33) Ishikawa, H.; Shiota, M.; Murakami, M.; Nakajima, I. Polymorphic Behavior of Palm Oil and Modified Palm Oils. Food Sci. Technol. Int., Tokyo 1997, 3, 77-81.

(34) Fredrick, E.; Van de Walle, D.; Walstra, P.; Zijtveld, J.; Fischer, S.; Van der Meeren, P.; Dewettinck, K. Isothermal crystallization behaviour of milk fat in bulk and emulsified state. Int. Dairy J. 2011, $21,685-695$.

(35) Hoerr, C. W. Morphology of fats, oils, and shortenings. J. Am. Oil Chem. Soc. 1960, 37, 539-546.

(36) X-ray diffraction by polycrystalline materials; Guinebretière, R., Ed.; Wiley: London, U.K., 2010; pp 127-153.

(37) Madsen, I. C.; Scarlett, N. V. Y.; Kern, A. Description and survey of methodologies for the determination of amorphous content via X-ray powder diffraction. Zeitschrift für Krist. 2011, 226, 944-955.

(38) Le Révérend, B. J. D.; Fryer, P. J.; Coles, S.; Bakalis, S. A Method to Qualify and Quantify the Crystalline State of Cocoa Butter in Industrial Chocolate. J. Am. Oil Chem. Soc. 2010, 87, 239-246.

(39) Gribnau, M. Determination of solid/liquid ratios of fats and oils by low-resolution pulsed NMR. Trends Food Sci. Technol. 1992, 3, 186-190.

(40) Lopez-Rubio, A.; Flanagan, B. M.; Gilbert, E. P.; Gidley, M. J. A novel approach for calculating starch crystallinity and its correlation with double helix content: A combined XRD and NMR study. Biopolymers 2008, 89, 761-768. 